\title{
ARBITRAGE WITH FRACTIONAL BROWNIAN MOTION
}

\author{
L. C. G. RogERS ${ }^{1}$ \\ School of Mathematical Sciences, University of Bath, Bath, United Kingdom
}

\begin{abstract}
Fractional Brownian motion has been suggested as a model for the movement of log share prices which would allow long-range dependence between returns on different days. While this is true, it also allows arbitrage opportunities, which we demonstrate both indirectly and by constructing such an arbitrage. Nonetheless, it is possible by looking at a process similar to the fractional Brownian motion to model long-range dependence of returns while avoiding arbitrage.
\end{abstract}

KEY WoRDS: fractional Brownian motion, Brownian motion, arbitrage, long-range dependence, equivalent martingale measure

\section{INTRODUCTION}

The log-Brownian model for the movement of share prices is widely used in the theory of mathematical finance, but empirically demonstrated to be incorrect in a number of ways. Various alternatives have been suggested to account for empirically observed deficiencies, among them the fractional Brownian motion, which displays dependence between returns on different days, in contrast to Brownian motion. However, as we will prove, the fractional Brownian motion is not a semimartingale (except in the Brownian case), and therefore there can be no equivalent martingale measure; by general results this means that there must be arbitrage. We then give a direct construction of arbitrage with fractional Brownian motion. While this may be the end of fractional Brownian motion as a model for the movement of the price of a share, it is not the end of all attempts to model a share price process with longrange dependence of returns. Formally, the fractional Brownian motion is the convolution of Brownian increments with a power-law kernel, and the arbitrage is happening because of the behavior of that kernel near zero. Long-range dependence is happening because of the behavior of the kernel at infinity, so the remedy is clear; we convolute the Brownian increments with some kernel which has the same behavior at infinity but a more orderly behavior at zero, and everyone will be happy! The conclusion shows one way in which this can be done.

To explain the problem in more detail, if $\left(W_{t}\right)_{t \in \mathbb{R}}$ is a standard Brownian motion with $W_{0}=0$ (so the paths of $W$ are continuous, and the increments of $W$ over disjoint intervals are independent zero-mean Gaussian random variables with variance equal to the length of the interval), then the fractional Brownian motion $\left(X_{t}\right)_{t \in \mathbb{R}}$ with self-similarity parameter

\footnotetext{
${ }^{1}$ Supported by EPSRC grant number GR/J82041. The author thanks the anonymous referee for carefully and perceptively reading this paper and suggesting several improvements.

Manuscript received March 1995; final revision received February 1996
} 
$H \in(0,1)$ is defined by ${ }^{2}$

$$
X_{t} \equiv k\left[\int_{-\infty}^{t}(t-s)^{H-1 / 2} d W_{s}-\int_{-\infty}^{0}(-s)^{H-1 / 2} d W_{s}\right] \text {, }
$$

where

$$
k^{-2} \equiv(2 H)^{-1}+\int_{0}^{\infty}\left((1+v)^{H-1 / 2}-v^{H-1 / 2}\right)^{2} d v .
$$

The process $X$ is clearly a zero-mean Gaussian process, and the constant $k$ is chosen to normalize the covariance structure neatly:

$$
E\left|X_{s}-X_{t}\right|^{2}=|t-s|^{2 H}
$$

The case $H=\frac{1}{2}$ corresponds to the familiar situation of Brownian motion, and $X$ is in general a self-similar process; for any $c>0$,

$$
(X(c t))_{t \in \mathbb{R}} \stackrel{\mathcal{D}}{=}\left(c^{H} X_{t}\right)_{t \in \mathbb{R}}
$$

At an intuitive level, it is easy to explain both of the results of this paper. First, from (1.2) we see that the increments process of $X$ is stationary and that $X_{t+\delta}-X_{t} \sim \delta^{H}$, which suggests that

$$
\sum_{j=1}^{2^{n}}\left|X\left(j 2^{-n}\right)-X\left((j-1) 2^{-n}\right)\right|^{p} \sim\left(2^{n}\right)^{1-p H} .
$$

Letting $n \rightarrow \infty$, we expect that the order- $p$ variation of $X$ will be infinite if $p<H^{-1}$, and zero if $p>H^{-1}$. This is only consistent with semimartingale behavior if $H=\frac{1}{2}$.

For the construction of arbitrage, we note that for $t>0$,

$$
E\left(X_{t} \mid \mathcal{G}_{0}\right)=\int_{-\infty}^{0}\left\{(t-s)^{H-1 / 2}-(-s)^{H-1 / 2}\right\} d W_{s},
$$

where $\mathcal{G}_{t} \equiv \sigma\left(\left\{W_{u}: u \leq t\right\}\right)$. If we knew that $E\left(X_{t} \mid \mathcal{G}_{0}\right)=E\left(X_{t} \mid \mathcal{F}_{0}\right)$, where $\mathcal{F}_{t} \equiv$ $\sigma\left(\left\{X_{u}: u \leq t\right\}\right)$, we may conclude from (1.5) that seeing $\left(X_{u}\right)_{u \leq 0}$ gives us information about the future behavior of $X$ (except in the case $H=\frac{1}{2}$ ), which can be exploited in the obvious way; if $E\left(X_{t} \mid \mathcal{F}_{0}\right)>0$, we would make a positive investment in the asset, but if $E\left(X_{t} \mid \mathcal{F}_{0}\right)<0$ we would short the asset. What remains is to show that we can carry out

\footnotetext{
${ }^{2}$ Strictly speaking, the two integrals on the right side of (1.1) cannot be defined separately, but the formally correct definition

$$
X_{t} \equiv k \int_{-\infty}^{\infty}\left\{\left((t-s)^{+}\right)^{H-1 / 2}-\left(s^{-}\right)^{H-1 / 2}\right\} d W_{s}
$$

does make good sense, even though it is less digestible than (1.1).
} 
the trading in a sufficiently controlled way to make arbitrage in finite time while ensuring that the wealth process will never fall below some fixed level. As the reader will see from Section 3, several of the steps involved require care; since $X$ is not a semimartingale, we have to rely on more elementary properties. Finally, in Section 4 we show how simple change-of-measure properties extend the conclusions of Sections 2 and 3 to more general Gaussian processes of the form $X_{t}=\int_{-\infty}^{t} a(t-s) d W_{s}$, where $a(t) \sim t^{H-1 / 2}(t \downarrow 0)$.

The first result of this paper has been known for some time; it certainly appears in the paper of Maheswaran and Sims (1993), and indeed was known to the present author in 1989 when the issue was drawn to his attention by Walter Willinger. Quite possibly other earlier references exist. ${ }^{3}$ However, since the fractional Brownian motion continues to appear as a model for an asset price process (for example, in Peters 1991 and Bouchaud and Sornette 1994), it seems that the problems in using it as an asset price process need to be more widely known. To amplify, the first result implies that for $H \neq \frac{1}{2}$ the process $X$ is not a semimartingale and therefore there can be no equivalent probability under which $X$ becomes a local martingale. This almost implies that there is arbitrage; Delbaen (1992) proved that the existence of an equivalent martingale measure is equivalent to the NFLVR (= no free lunch with vanishing risk) condition. This condition is more restrictive than the condition that there is no arbitrage and, indeed, Delbaen and Schachermayer (1994) and Back and Pliska (1991) give examples where the condition holds, but the NA (= noarbitrage) NFLVR condition does not. To be exact, we say that an arbitrage exists if there is some trading strategy whose gains process $\left(\xi_{t}\right)_{0 \leq t \leq 1}$ satisfies (i) $\xi_{0}=0 \leq \xi_{1}$, (ii) $\xi_{t} \geq-1$ for all $0 \leq t \leq 1$, and (iii) $P\left(\xi_{1}>0\right)>0$. It is immediate that the existence of an arbitrage in this strict sense implies existence of a FLVR, but the converse is false. Maheswaran and Sims describe a trading strategy which by time 1 can create a wealth whose mean is at least 1 and whose variance is as small as one wishes, but this fails to be an arbitrage because condition (ii) fails, and this really matters, since this is the condition which prevents arbitrage by double-or-quits strategies played on Brownian motion. Delbaen and Schachermeyer (1994, Theorem 7.2) give a proof in a general setting that if a process $X$ is not a semimartingale, there must exist a FLVR. Not surprisingly, with the specific structure of a fractional Brownian motion, we can do better; there does exist an arbitrage in the strict sense defined above and the somewhat delicate task of constructing it is settled in Section 3.

\section{FRACTIONAL BROWNIAN MOTION IS A SEMIMARTINGALE ONLY IF $H=\frac{1}{2}$}

The title of this section is its main result, and the proof follows the lines sketched in the Introduction. Fix the parameter $H$ of the fractional Brownian motion, and consider for $p>0$ fixed

$$
Y_{n, p} \equiv \sum_{j=1}^{2^{n}}\left|X\left(j 2^{-n}\right)-X\left((j-1) 2^{-n}\right)\right|^{p}\left(2^{n}\right)^{p H-1} .
$$

It is clear that $E Y_{n, p}=E\left|W_{1}\right|^{p}$, the same for all $n$, and it is even true that $\left\|Y_{n, p}\right\|_{2}$ remains bounded for all $n$. If we now consider

$$
\tilde{Y}_{n, p} \equiv 2^{-n} \sum_{j=1}^{n}\left|X_{j}-X_{j-1}\right|^{p},
$$

\footnotetext{
${ }^{3}$ A. N. Shiryayev tells me that it appears as an example in his 1986 book (in Russian) with R. S. Liptser Theory of Martingales.
} 
this has (for each $n$ ) the same law as $Y_{n, p}$. Noticing that the sequence $\left(X_{k}-X_{k-1}\right)_{k \in \mathbb{Z}}$ is stationary and ergodic, the ergodic theorem tells us that

$$
\tilde{Y}_{n, p} \rightarrow E\left|X_{1}-X_{0}\right|^{p} \equiv c_{p} \quad(n \rightarrow \infty)
$$

almost surely and in $L^{1}$. Hence

$$
Y_{n, p} \stackrel{\mathcal{D}}{\rightarrow} c_{p} \quad(n \rightarrow \infty)
$$

and therefore $Y_{n, p} \stackrel{P}{\rightarrow} c_{p}$. Hence,

$$
V_{n, p} \equiv \sum_{j=1}^{2^{n}}\left|X\left(j 2^{-n}\right)-X\left((j-1) 2^{-n}\right)\right|^{p} \stackrel{P}{\rightarrow} \begin{cases}0 & \text { if } \quad p H>1 \\ +\infty & \text { if } \quad p H<1\end{cases}
$$

If $H>\frac{1}{2}$, we can choose $p \in\left(H^{-1}, 2\right)$ such that $V_{n, p} \rightarrow 0$ in probability, and therefore almost surely down a fast subsequence. This implies that the quadratic variation of $X$ is zero, and so (if $X$ were to be a semimartingale) $X$ must be a finite-variation process. But since for $p \in\left(1, H^{-1}\right), V_{p} \equiv \lim _{n \rightarrow \infty} V_{n, p}$ is almost surely infinite, and (by scaling) the order- $p$ variation on any interval is infinite almost surely, $X$ cannot be finite variation. If $H<\frac{1}{2}$, we can choose $p>2$ such that $p H<1$, and the order- $p$ variation of $X$ on $[0,1]$ (and hence on any fixed interval) must be infinite. This contradicts the almost-sure finiteness of the quadratic variation of $X$, assuming $X$ is a semimartingale. Either way, if $H \neq \frac{1}{2}, X$ is not a semimartingale.

\section{MAKING AN ARBITRAGE}

As we indicated in the Introduction, there is a priori a difference between $\mathcal{G}_{t} \equiv \sigma\left(\left\{W_{s}\right.\right.$ : $s \leq t\})$ and $\mathcal{F}_{t} \equiv \sigma\left(\left\{X_{s}: s \leq t\right\}\right)$ (though $\mathcal{F}_{t} \subseteq \mathcal{G}_{t}$ is obvious), which might matter for the construction of arbitrage; if our arbitrage-generating investment policy (which will be of a simple "buy-and-hold" nature) were adapted to $\left(\mathcal{G}_{t}\right)$, would there necessarily be an $\left(\mathcal{F}_{t}\right)$-adapted arbitrage-generating policy? As it turns out, our construction of the arbitrage need only see the smaller filtration $\left(\mathcal{F}_{t}\right)$, but for its passing interest we record here a result which shows that the two filtrations are the same.

Proposition 3.1. For any $a>0$,

$$
\begin{aligned}
E\left[X_{a} \mid X_{u}: u \leq 0\right] & =\int_{-\infty}^{0} \frac{|t / a|^{-H-1 / 2}}{a+|t|} X_{t} \frac{H-1 / 2}{\Gamma\left(\frac{1}{2}+H\right) \Gamma\left(\frac{3}{2}-H\right)} d t \\
& =\int_{-\infty}^{0}\left\{(a-s)^{H-1 / 2}-(-s)^{H-1 / 2}\right\} d W_{s}
\end{aligned}
$$

In particular, $\left(\mathcal{G}_{t}\right)=\left(\mathcal{F}_{t}\right)$ to within null sets. 
We omit details of the proof; to prove (3.1) we take the integral representation (1.1) of $X$ and perform a change of order of integration to show it is the same as (3.2). Since (3.2) is $E\left[X_{a} \mid W_{u}: u \leq 0\right]$, that proves all but the last assertion, and for the last assertion we find that the process $\left(\int_{-\infty}^{0}(a-s)^{-c} d W_{s}\right)_{a>0}$ is $\mathcal{F}_{0}$ measurable for $c=H-\frac{5}{2}$ (by differentiating twice), and $W$ can be recovered from this Mellin transform.

So now our task is to construct an arbitrage. We are going to split $(-\infty, 0)$ into $\bigcup_{n \in \mathbb{Z}}\left(-2^{-n+1},-2^{-n}\right]$ and only trade in the share during intervals which look promising. During such an interval, if the gain of our investment in the share gets too high or too low, we immediately sell it and wait until the end of the interval, thus ensuring bounded gains on each time interval. The main task is to show that there do exist time intervals which look promising; that there are plenty of them follows by stationarity.

LEMMA 3.1. Suppose that $\left(\Omega, \mathcal{F},\left(\mathcal{F}_{t}\right)_{t \in \mathbb{R}^{+}}, P\right)$ is a filtered probability space and $\left(X_{t}\right)_{t \in \mathbb{R}^{+}}$ is a continuous adapted process, with $X_{0} \in L^{1}(P)$. For any real $a<b$ and $0 \leq t \leq c$ define

$$
\tau(t, c ; a, b) \equiv \inf \left\{u>t: X_{u} \notin[a, b]\right\} \wedge c .
$$

Suppose that for all rational $a, b, c, q$, for which $q<c$ and $a<b$, we have

$$
E\left(X_{\tau(q, c ; a, b)} \mid \mathcal{F}_{q}\right)=X_{q} \text { a.s. }
$$

then $X$ is a local martingale.

Proof. First note that if $T \leq c$ is any stopping time, then the equality (3.4) is easily extended to

$$
E\left(X_{\tau(T, c ; a, b)} \mid \mathcal{F}_{T}\right)=X_{T} \text { a.s. },
$$

by approximating $T$ by $T^{(n)} \equiv 2^{-n}\left(\left[2^{n} T\right]+1\right)$, a sequence of stopping times taking discretely many rational values and decreasing to $T$.

Now fix $N \in \mathbb{N}$, and define $\zeta \equiv \tau(0, N ;-N, N)$. We shall prove that $X(t \wedge \zeta)$ is a martingale, and this will be sufficient. To this end, fix $\varepsilon^{-1} \in \mathbb{N}$ and define the stopping times $\sigma_{n}^{\varepsilon}$ by $\sigma_{0}^{\varepsilon}=0$,

$$
\sigma_{n+1}^{\varepsilon} \equiv \inf \left\{u>\sigma_{n}^{\varepsilon}: X_{u} \neq X\left(\sigma_{n}^{\varepsilon}\right), X_{u} \in \varepsilon \mathbb{Z}\right\} \wedge \zeta
$$

for $n \geq 0$. Evidently $\sigma_{n}^{\varepsilon} \uparrow \zeta$ as $n \uparrow \infty$. It is also clear that $\left(X\left(\sigma_{n}^{\varepsilon}\right), \mathcal{F}\left(\sigma_{n}^{\varepsilon}\right)\right)_{n \geq 0}$ is a martingale, using (3.4). Since $\left|X\left(\sigma_{n}^{\varepsilon}\right)\right| \leq N \vee\left|X_{0}\right|$ for all $n$, we have that for all $n$,

$$
X\left(\sigma_{n}^{\varepsilon}\right)=E\left[X_{\zeta} \mid \mathcal{F}\left(\sigma_{n}^{\varepsilon}\right)\right],
$$

and from this it follows easily that for all $t<N$,

$$
X_{t \wedge \zeta}=E\left[X_{\zeta} \mid \mathcal{F}_{t}\right]
$$


Now we know that (unless $H=\frac{1}{2}$ ) the fractional Brownian motion $X$ is not a local martingale; indeed, if $H<\frac{1}{2}$ we have from Section 2 that for some $p>2$ the order- $p$ variation of $X$ is infinite over every interval of positive length (which is not consistent with the locally finite order- 2 variation of a continuous local martingale), and if, on the other hand, $H>\frac{1}{2}$, we shall have for some $p<2$ that the order- $p$ variation of $X$ is zero, which would imply that the order- 2 variation of $X$ is zero and, therefore, that the continuous local martingale $X$ is constant.

Thus, we know that the condition (3.4) of Lemma 3.1 must fail for some rational $q<c$ and $a<b$;

$$
P\left[E\left(X_{\tau(q, c ; a, b)} \mid \mathcal{F}_{q}\right) \neq X_{q}\right]>0 .
$$

This is not quite in the form we want for later use. Define for $0 \leq t \leq c$ and for $\alpha<0<\beta$ the stopping times

$$
\rho(t, c ; \alpha, \beta) \equiv \inf \left\{u>t ; X_{u}-X_{t} \notin[\alpha, \beta]\right\} \wedge c .
$$

LEMMA 3.2. If $\left(X_{t}\right)_{t \in \mathbb{R}}$ is fractional Brownian motion, $H \neq \frac{1}{2}$, then for some $\alpha<0<\beta$ and $c>0$

$$
P\left[E\left(X_{\rho(0, c ; \alpha, \beta)} \mid \mathcal{F}_{0}\right) \neq X_{0}\right]>0 .
$$

Proof. Suppose that the result were false; for all $\alpha<0<\beta$ and $q<c E\left(X_{\rho(q, c ; \alpha, \beta)} \mid \mathcal{F}_{q}\right)$ $=X_{q}$ almost surely. This contradicts (3.4), which we can see as follows. With $q, c, a, b$ being now values for which (3.4) holds, we shall construct stopping times $T_{n} \downarrow \tau(q, c ; a, b)$ with the property that $X-X_{q}$ is bounded on $\left[q, T_{n}\right]$ for each $n$, and $E\left(X\left(T_{n}\right) \mid \mathcal{F}_{q}\right)=X_{q}$ for every $n$. Take $\delta \equiv b-a$ and define

$$
\begin{aligned}
T_{n} & =q \quad \text { if } X_{q} \notin(a, b] \\
& =\rho(q, c ;-j \delta / n,(n-j+1) \delta / n) \quad \text { if }(j-1) \delta n^{-1}<X_{q}-a \leq j \delta n^{-1}
\end{aligned}
$$

for $j=1, \ldots, n$. It is not hard to verify that the $T_{n}$ have the properties claimed and, if (3.7) were to fail, then $E\left(X\left(T_{n}\right) \mid \mathcal{F}_{q}\right)=0$ for all $n$. This would ensure that (3.4) fails, and we know this is not correct.

Let us now see how to use Lemma 3.2 to make arbitrage. We shall make arbitrage by investing suitably in $X$ during the time interval $[-1,0)$. For each $n \in \mathbb{Z}$ define the process

$$
Y_{n}(t) \equiv\left\{X\left(-2^{-n}(2-t)\right)-X\left(-2^{-n+1}\right)\right\} 2^{n H} \quad(0 \leq t \leq 1) .
$$

By the scaling properties of $X$ the sequence $\left(Y_{n}\right)_{n \in \mathbb{Z}}$ of $C([0,1])$-valued random variables is stationary, and even ergodic because $\bigcap_{n} \sigma\left(Y_{k}: k \leq-n\right)$ is contained in the tail $\sigma$-field of $W_{-t}$, which is trivial. If we let $\mathcal{G}_{n} \equiv \mathcal{F}\left(-2^{-n}\right)$, then $\left(Y_{n}\right)$ is adapted to $\mathcal{G}_{n}$ and because 
of Lemma 3.2 and scaling properties, there exist $\alpha<0<\beta$ and $\varepsilon>0$ such that

$$
P\left[E\left[Y_{n}\left(\tau_{n}\right) \mid \mathcal{G}_{n-1}\right] \geq \varepsilon\right] \geq \varepsilon
$$

where

$$
\tau_{n} \equiv \inf \left\{t: Y_{n}(t) \notin[\alpha, \beta]\right\} \wedge 1
$$

In view of this the ergodic theorem guarantees that

$$
P\left[E\left(Y_{n}\left(\tau_{n}\right) \mid \mathcal{G}_{n-1}\right) \geq \varepsilon \quad \text { for infinitely many } n \geq 0\right]=1 .
$$

Call the time interval $\left(-2^{-n+1},-2^{-n}\right]$ period $n$, and say that period $n$ is promising if $E\left(Y\left(\tau_{n}\right) \mid \mathcal{G}_{n-1}\right) \geq \varepsilon$. Since there will be infinitely many promising periods, we may describe our investment strategy by saying that (at least to begin with) we invest unit amount in each promising period, but should $Y_{n}$ leave $[\alpha, \beta]$ during the promising period we immediately sell our holding and wait until the end of the period. Thus the gain made during a promising period is always bounded and has positive mean. Let $\eta_{n}$ denote the accumulated gain by the end of period $n$. Since $\alpha \leq \eta_{n}-\eta_{n-1} \leq \beta$, and $E\left(\eta_{n}-\eta_{n-1} \mid \mathcal{G}_{n-1}\right) \geq \varepsilon$, we may pick $\lambda>0$ so small that for all $n$,

$$
E\left[e^{-\lambda \eta_{n}} \mid \mathcal{G}_{n-1}\right] \leq e^{-\lambda \eta_{n-1}}
$$

(Of course, if period $n$ is not promising, $\eta_{n}=\eta_{n-1}$ !) Thus $e^{-\lambda \eta_{n}}$ is a nonnegative supermartingale, convergent almost surely to 0 (since there are infinitely many promising periods). If we stop $\eta$ at the first time $v$ that $\eta<\alpha$, then

$$
P(v<\infty) \leq \exp (\lambda \alpha)=1-\theta<1
$$

and on the event $\{v=+\infty\}, \eta_{n} \rightarrow \infty$. Now we can describe fully how we make arbitrage. Invest unit amount ${ }^{4}$ in each promising period until either $\eta$ has risen to 1 or $\eta$ falls to below $\alpha$. The former happens with probability at least $\theta$ (and results in our gain of 1), while if the latter happens we shall have lost at most $2|\alpha|$. If the latter happens proceed to invest $\frac{1}{2}$ in each promising period until either the accumulated gain $\eta$ has risen to 1 or the accumulated gain has fallen below $5 \alpha / 2$. If the latter happens, we shall have lost at most $3|\alpha|$, and we proceed to invest $\frac{1}{4}$ in each promising period until either $\eta$ has risen to 1 or $\eta$ has fallen below $13 \alpha / 4$. If we continue in this way, successively halving our stake when things go badly, we shall eventually be successful and make net gain at least 1 , and the worst that can happen is that our wealth meantime could fall to $4 \alpha$.

\footnotetext{
${ }^{4}$ That is, unit amount in $Y$, which is the same as investing amount $2^{n H}$ in $X$ during period $n$.
} 


\section{EXTENDING TO GAUSSIAN PROCESSES SIMILAR TO FRACTIONAL BROWNIAN MOTION}

In this section we use routine change-of-measure arguments to show that if we convolute Brownian motion with a kernel which is similar at zero (in a sense to be made precise) to the kernel used to make fractional Brownian motion, then the existence of arbitrage persists. In contrast, we show in Section 5 that we can smooth out the kernel at zero, remove the arbitrage, but keep long-range dependence.

In Section 3 we created arbitrage by trading on the fractional Brownian motion only during the interval $[-1,0)$, but we could just as well have made arbitrage in $[0,1)$ by shifting the strategy by one unit of time to the right. The strategy so shifted will (with probability 1 ) make net gain 1 with a lower bound on the wealth process throughout $[0,1]$; so if we change to an equivalent measure, the same will be true, almost surely with respect to the equivalent measure.

Let us write

$$
\varphi(s) \equiv s^{H-1 / 2} I_{\{s>0\}} \equiv \varphi_{0}(s)+\varphi_{1}(s),
$$

where $\varphi_{0}, \varphi_{1}$ are nonnegative, with the properties

$\varphi_{1}$ is $C_{b}^{3}(\mathbb{R})$, with support in $(1, \infty)$,

$$
\varphi_{0} \text { is compactly supported in [0,2]. }
$$

Now define

$$
X_{t}^{i} \equiv \int_{-\infty}^{t} \varphi_{i}(t-s) d W_{s}-\int_{-\infty}^{0} \varphi_{i}(-s) d W_{s},
$$

and observe that we may integrate by parts to express $X^{1}$ as

$$
X_{t}^{1}=\int_{-\infty}^{t}\left\{\varphi_{1}^{\prime}(t-s)-\varphi_{1}^{\prime}(-s)\right\} W_{s} d s
$$

From this, it is not hard to check that $X^{1}$ is differentiable, with

$$
\frac{d}{d t} X_{t}^{1}=\int_{-\infty}^{t} \varphi_{1}^{\prime \prime}(t-s) W_{s} d s \quad(t \geq 0) .
$$

Next we aim to identify a process $\mu$ which will be nonzero only in $[0,1]$ with the property that for $0 \leq t \leq 1$,

$$
X_{t}^{1}=\int_{0}^{t} \varphi_{0}(t-s) \mu_{s} d s,
$$

this last integral being equal also to $\int_{0}^{t} \varphi(t-s) \mu_{s} d s$ for $0 \leq t \leq 1$. If $f$ is $C^{2}$ with compact support, $f=X^{1}$ on $[0,1]$, we can solve the convolution equation (4.4) $f=\varphi * \mu$ by taking 
the Laplace transforms (denoted by a tilde) to obtain

$$
\tilde{f}(\lambda)=\tilde{\varphi}(\lambda) \tilde{\mu}(\lambda)=\lambda^{-H-1 / 2} \Gamma\left(H+\frac{1}{2}\right) \tilde{\mu}(\lambda),
$$

yielding

$$
\tilde{\mu}(\lambda)=\frac{1}{\Gamma\left(H+\frac{1}{2}\right)} \lambda^{H-3 / 2} \lambda^{2} \tilde{f}(\lambda) .
$$

Now since $\lambda^{H-3 / 2}$ is the Laplace transform of $t^{3 / 2-H} / \Gamma\left(\frac{5}{2}-H\right)$, and $\lambda^{2} \tilde{f}(\lambda)$ is the Laplace transform of the measure $f^{\prime \prime}(t) d t+f^{\prime}(0) \delta_{0}(d t)$, we conclude from (4.6) that

$$
\mu_{t}=\left\{\int_{0}^{t}(t-s)^{3 / 2-H} f^{\prime \prime}(s) d s+f^{\prime}(0) t^{3 / 2-H}\right\} / \Gamma\left(\frac{5}{2}-H\right) \Gamma\left(H+\frac{1}{2}\right) .
$$

Observe that, because $\varphi_{1}$ is supported in $(1, \infty)$, it follows that $X_{t}^{1}$ is $\mathcal{F}_{0}$-measurable for all $t \in[0,1]$, and hence the process $\left(\mu_{t}\right)_{0 \leq t \leq 1}$ is again $\mathcal{F}_{0}$-measurable. We have then for $t \in[0,1]$,

$$
\begin{aligned}
X_{t} & =X_{t}^{0}+X_{t}^{1} \\
& =\int_{-\infty}^{t} \varphi_{0}(t-s) d W_{s}-\int_{-\infty}^{0} \varphi_{0}(-s) d W_{s}+\int_{0}^{t} \varphi_{0}(t-s) \mu_{s} d s \\
& =\int_{-\infty}^{t} \varphi_{0}(t-s)\left\{d W_{s}+\mu_{s} d s\right\}-\int_{-2}^{0} \varphi_{0}(-s) d W_{s} .
\end{aligned}
$$

Since the process $\mu$ is continuous and $\mathcal{F}_{0}$-measurable, the drifting Brownian motion $d \tilde{W}_{t} \equiv$ $d W_{t}+\mu_{t} d t$ is equivalent to Brownian motion, and so from (4.6), since arbitrage is possible with $X$, it must also be possible with $X^{0}$.

The process $X^{0}$ comes from convoluting $d W$ with a function $\varphi_{0}$ which is equal to $\varphi$ in a neighborhood of zero, but would we still get arbitrage if we convolute $d W$ with $\tilde{\varphi}_{0}$, where $\tilde{\varphi}_{0}$ is like $\varphi$ in a neighborhood of zero? We do. Suppose that we express $\varphi_{0}=\tilde{\varphi}_{0}+\tilde{\varphi}_{1}$, where $\tilde{\varphi}_{1} \in C^{3}(\mathbb{R})$, with support in $[0,2]$, and $\tilde{\varphi}_{1}^{\prime}(0)=0$. The argument is similar to that given above; we seek a process $\left(\tilde{\mu}_{t}\right)_{0 \leq t \leq 1}$ such that

$$
\int_{-\infty}^{t} \varphi_{0}(t-s)\left(d W_{s}+\tilde{\mu}_{s} d s\right)=\int_{-\infty}^{t} \tilde{\varphi}_{0}(t-s) d W_{s}+\xi
$$

where $\xi$ is some unimportant $\mathcal{F}_{0}$-measurable random variable. Provided $\tilde{\mu}$ has enough integrability, the process on the left of (4.9) is equivalent to $X^{0}$, so an arbitrage can be 
constructed for the process on the right of (4.9). We need to pick $\tilde{\mu}$ so that

$$
\begin{aligned}
\int_{-\infty}^{t} \varphi_{0}(t-s) \tilde{\mu}_{s} d s & =\int_{-\infty}^{t}\left\{\tilde{\varphi}_{1}(t-s)-\tilde{\varphi}_{1}(-s)\right\} d W_{s}+\int_{-\infty}^{0} \varphi_{0}(-s) \tilde{\mu}_{s} d s \\
& =\int_{-\infty}^{t}\left\{\tilde{\varphi}_{1}^{\prime}(t-s)-\tilde{\varphi}_{1}^{\prime}(-s)\right\} W_{s} d s+\int_{-\infty}^{0} \varphi_{0}(-s) \tilde{\mu}_{s} d s .
\end{aligned}
$$

As before, the recipe (4.7) can be used to obtain $\tilde{\mu}$, which is now an adapted Gaussian process, vanishing at zero. Can it be used to change measure? We can use the Novikov criterion (see, for example, Ikeda and Watanabe 1981) to guarantee that a change of measure exists at least up to some positive time $\varepsilon$. This will be enough, because an arbitrage could have been constructed by that time.

\section{CONCLUSION}

We have seen that fractional Brownian motion is an absurd candidate for a log-price process. We have also seen that the arbitrage is arising because of the behavior of the kernel $\varphi(t)=$ $t^{H-1 / 2} I_{\{t>0\}}$ on small time scales. Since the fractional Brownian motion was introduced to model perceived long-range dependence in share returns, the way around all the problems is obvious; if we define

$$
X_{t}=\int_{-\infty}^{t} \varphi(t-s) d W_{s}-\int_{-\infty}^{0} \varphi(-s) d W_{s}
$$

where $\varphi \in C^{2}(\mathbb{R}), \varphi(0)=1, \varphi^{\prime}(0)=0$, and $\lim _{t \rightarrow \infty} \varphi^{\prime \prime}(t) t^{5 / 2-H}$ exists in $(0, \infty)$, then $X$ is a Gaussian process, with the same long-range dependence as fractional Brownian motion and yet (integrating by parts)

$$
\begin{aligned}
X_{t} & =W_{t}+\int_{-\infty}^{t} \varphi^{\prime}(t-s) W_{s} d s-\int_{-\infty}^{0} \varphi^{\prime}(-s) W_{s} d s \\
& =W_{t}+\int_{0}^{t}\left(\int_{-\infty}^{s} \varphi^{\prime \prime}(s-v) W_{v} d v\right) d s,
\end{aligned}
$$

exhibiting $X$ as a semimartingale. We could take

$$
\varphi(t)=\left(\varepsilon+t^{2}\right)^{(2 H-1) / 4},
$$

for example.

\section{REFERENCES}

BACK, K., and S. PlisKa (1991): "On the Fundamental Theorem of Asset Pricing with an Infinite State Space," J. Math. Econ., 20, 1-18. 
Bouchaud, J.-P, and D. SornetTe (1994): "The Black-Scholes Option Pricing Problem in Mathematical Finance: Generalization and Extensions for a Large Class of Stochastic Proceses," J. Phys. France, 4, 863-881.

Delbaen, F. (1992): "Representing Martingale Measure When Asset Prices Are Continuous and Bounded," Math. Finance, 2, 107-130.

DELbAEN, F., and W. SchaCHERMAYER (1994): "A General Version of the Fundamental Theorem of Asset Pricing," Math. Ann., 300, 463-520.

IKeDA, N., and S. Watanabe (1981): Stochastic Differential Equations and Diffusion Processes. Amsterdam: North-Holland; Tokyo: Kodansha.

MAHESWARAN, S., and C. A. SIMS (1993): "Empirical Implications of Arbitrage-Free Asset Markets," in Models, Methods and Applications of Econometrics: Essays in Honor of A. R. Bergstrom, ed. P. C. B. Phillips. Cambridge, MA: Blackwell.

PETERS, E. E. (1991): Chaos and Order in Capital Markets. New York: Wiley. 